\title{
PSICODIAGNÓSTICO INTERVENTIVO PSICANALÍTICO
}

sicóloga, doutora em Medicina (Saúde Mental) pela Universidade de São Paulo (2006). Professora Titular do Centro de Universitário Cesumar. Coordena o Grupo de Pesquisas em Saúde Mental e Desenvolvimento na Infância e Adolescência Cesumar/CNPq. Docente do curso de Mestrado em Tecnologias Limpas pela Unicesumar.

Mercês Maria Tomael Psicóloga clínica, especialista em psicoterapia psicanalítica e em psicopedagogia.

Bruna Rafaele Milhorini Greinert Acadêmica do curso de Psicologia do Centro Universitário Cesumar e pesquisadora PIBIC pelo CNPq.

\section{Resumo}

O psicodiagnóstico interventivo trouxe modificações no processo de avaliação clínica, principalmente no que se refere à atitude e às intervenções do profissional durante o procedimento. O objetivo deste artigo é, a partir de um estudo teórico psicanalítico, descrever os resultados obtidos em pesquisas com pacientes submetidos ao psicodiagnóstico interventivo, tendo em vista a necessidade de conhecer as especificidades práticas e teóricas da aplicação deste método. Os estudos foram selecionados por meio de bases de dados eletrônicas, tais como: LILACS, Scielo e BVS-PSI, e bibliotecas universitárias. Na análise dos artigos verificou-se que os pacientes atendidos se beneficiaram com o psicodiagnóstico interventivo, possibilitando-Ihes pensar o seu trajeto enquanto sujeito de sua história. Constatou-se que o psicodiagnóstico interventivo psicanalítico é um método que permite a compreensão intuitiva do inconsciente, em que o paciente tem a possibilidade de, a partir de um holding bem estabelecido, num ambiente suficientemente bom, diminuir seu sofrimento mental.

Palavras-chave: avaliação da personalidade; processo psicoterapêutico; psicologia clínica; clínica psicanalítica.

\section{PSYCHOANALYTIC INTERVENTIVE PSYCHODIAGNOSIS}

\begin{abstract}
Interventive psychodiagnosis introduced changes in the clinical evaluation process mainly with regard to the stance and the interventions of the professional during procedures. Current paper registers the results obtained in research work with patients undergoing interventive psychodiagnosis, from a theoretical psychoanalytic investigation, so that the practical and theoretical specificities for the application of this method could be known. Studies were retrieved from electronic databases such as LILACS, Scielo, BVS-PSI and university libraries. The articles showed that patients attended to were benefitted by interventive psychodiagnosis, making it possible to construct their trajectory as agents of their own history. Psychoanalytic interventive psychodiagnosis is a method that allows the intuitive understanding of the unconscious through which the patients have the possibility of decreasing their mental suffering by a well-established holding within an adequate environment.
\end{abstract}

Keywords: personality assessment; psychotherapeutic process; clinical psychology; psychoanalytic clinic. 


\title{
EL PSICODIAGNÓSTICO INTERVENCIONISTA PSICOANALÍTICO
}

\begin{abstract}
Resumen
El psicodiagnóstico intervencionista trajo cambios en el proceso de evaluación clínica, mayormente en lo referente a la postura y a las intervenciones del profesional durante el procedimiento. El objetivo de este artículo es, a partir de un estudio teórico psicoanalítico, describir los resultados obtenidos en investigaciones con pacientes sometidos al psicodiagnóstico intervencionista, considerando las especificidades prácticas y teóricas de la aplicación de este método. Los estudios fueron seleccionados por medio de bases de datos electrónicas, tales como: LILACS, Scielo y BVS-PSI, y en bibliotecas universitarias. En el análisis de los artículos se verificó que los pacientes atendidos se beneficiaron con el psicodiagnóstico intervencionista, posibilitándoles pensar su trayecto como sujeto de su historia. Se constató que el psicodiagnóstico intervencionista psicoanalítico es un método que permite la comprensión intuitiva del inconsciente, en que el paciente tiene la posibilidad de, a partir de un holding bien establecido, en un ambiente suficientemente bueno, disminuir su sufrimiento mental.

Palabras clave: evaluación de la personalidade; proceso psicoterapêutico; psicología clínica; clínica psicoanalítica.
\end{abstract}

\section{INTRODUÇÃO}

O psicodiagnóstico interventivo se configura como um processo de investigação diagnóstica, que inclui simultaneamente intervenções as quais poderão trazer mudanças e bem estar para o paciente desde as consultas iniciais. A aplicação do psicodiagnóstico interventivo vem sendo utilizada desde a década de 90, por alguns profissionais da área de psicologia. Em alguns atendimentos foram constatadas mudanças nos pacientes após serem submetidos ao processo psicodiagnóstico sem a intenção de interferência. Esses acontecimentos levaram os profissionais a questionar, se simplesmente o fato do contato paciente-terapeuta, já provocaria situações que propiciariam a reorganização mental do paciente.

Desde então, alguns psicólogos se interessaram pelo assunto e, nas últimas décadas, começaram a surgir estudos a respeito desse tipo de psicodiagnóstico. Winnicott (1971) citado por Silva (2010) escreveu sobre os resultados positivos obtidos com a interpretação da fala dos pacientes nas primeiras consultas. Walter Trinca (1998) formulou o psicodiagnóstico compreensivo, técnica em que o terapeuta interage com o paciente de forma empática e obtém um conhecimento profundo sobre seu funcionamento mental. Nos últimos anos, cada vez mais, os profissionais da psicanálise se aproximam de seus pacientes tentando abstrair aspectos de suas personalidades.

Winnicott (1984) foi o precursor desse tipo de avaliação, denominada por ele de "Consultas Terapêuticas", momento em que fazia o tratamento com 
poucas entrevistas, na maioria dos casos em apenas uma, utilizando-se de interpretações no momento em que sentia o paciente receptivo para isso. Para o autor, nas consultas terapêuticas, o relacionamento entre terapeuta e paciente, deve assegurar um clima de confiança para suprir a expectativa do indivíduo que está em processo psicodiagnóstico. Com isso se estabelece uma identificação mútua, entre terapeuta e paciente, parecida àquela da relação mãe/bebê, no início do desenvolvimento.

Segundo Winnicott (1984) citado por Barbieri, Jacquemin e Alves (2004; 2007) é plenamente possível fazer "um pequeno tratamento" durante as entrevistas iniciais e que é nesse momento que aparecem informações que só poderiam reaparecer meses ou anos mais tarde em uma psicoterapia.

Os precursores do psicodiagnóstico interventivo no Brasil foram os profissionais da abordagem fenomenológico-existencial. Ancona-Lopez (1998) e Santiago (1998) percebiam que, independentemente da vontade do terapeuta, os pacientes mostravam melhoras durante o processo psicodiagnóstico, e começaram, então, a investigar esses acontecimentos.

Para Barbieri (2009), o psicodiagnóstico interventivo é "um procedimento clínico que consiste em efetuar intervenções já no momento de realização de entrevistas e aplicação de testes, oferecendo ao paciente, devoluções durante todo o processo avaliativo e não somente ao seu final" (p. 02). Nessa perspectiva de psicodiagnóstico, os testes configuram-se como instrumentos que facilitam o contato do psicólogo com o paciente e auxiliam nas intervenções durante o processo psicodiagnóstico como consequência daquilo que o paciente apresenta, de forma a provocar mudanças (Paulo, 2009).

No psicodiagnóstico interventivo não há uma organização sequencial, com passos a serem seguidos, e o número de sessões não é predeterminado. Além disso, o profissional psicanalítico, como 'objeto subjetivo' deve proporcionar ao paciente a possibilidade, por meio da vivência emocional, de retomar seu desenvolvimento. É de extrema importância esse profissional ser visto e sentido como aquele que pode ajudar (Barbieri, 2010).

O psicodiagnóstico interventivo de orientação psicanalítica começou a ganhar força no Brasil somente a partir do ano 2000. Esse tipo de psicodiagnóstico inova o modelo tradicional e permite ao profissional construir uma visão compreensiva do paciente, ao mesmo tempo em que poderá ser um processo gerador de mudanças. 
Estudos sobre a influência do psicodiagnóstico interventivo na organização mental do paciente apresentam subsídios que reforçam a utilização desse modelo de avaliação (Paulo, 2006; Tardivo, 2007; Barbieri, 2008). Entretanto, permanecem alguns questionamentos, como por exemplo, de que maneira o psicodiagnóstico interventivo pode ser empregado na área clínica, tendo em vista a necessidade de conhecer as especificidades práticas e teóricas da aplicação deste método. Portanto, o objetivo deste artigo é, a partir de um estudo teórico sob o referencial psicanalítico, descrever os resultados obtidos em pesquisas com pacientes submetidos ao psicodiagnóstico interventivo. Deste modo, esta pesquisa busca auxiliar o profissional da psicologia que procura novas ferramentas em sua atuação clínica sob o referencial psicanalítico, visando fortalecer a atividade avaliativa e interpretativa.

\section{MÉTODO}

O presente trabalho se caracteriza como um estudo teórico psicológico. As fontes de consulta foram livros, periódicos e artigos científicos, selecionados por meio de bases de dados eletrônicas, tais como: LILACS, Scielo e BVS-PSI, e bibliotecas universitárias. Procedeu-se à seleção dos artigos definindo-se como período de consulta os anos de 2002 a 2012 e utilizando-se como descritores: psicodiagnóstico interventivo e psicanálise. Excluíram-se os artigos que investigavam o psicodiagnóstico interventivo por meio de outra abordagem psicológica. Com base nestes critérios foram identificados nove artigos.

$\mathrm{O}$ artigo foi dividido em três categorias principais. A primeira contemplou a utilização do psicodiagnóstico interventivo no atendimento a crianças, de forma a entender como este instrumento pode contribuir para o processo psicoterapêutico infantil. Em seguida, foi abordado o psicodiagnóstico interventivo realizado no atendimento de adultos e os benefícios terapêuticos que pode proporcionar ao paciente desde as consultas iniciais, visando à diminuição de seu sofrimento psíquico. A terceira categoria abordou o psicodiagnóstico interventivo psicanalítico e a formação profissional do psicólogo. Pretendeu-se, assim, refletir sobre o aprendizado desta nova modalidade de psicodiagnóstico ainda na graduação, pois esta ferramenta visa auxiliar o acadêmico em formação na obtenção da compreensão diagnóstica do paciente. 


\section{RESULTADOS}

De um modo geral, o conjunto de artigos analisados evidenciou o interesse pelo psicodiagnóstico na infância. Dos nove artigos relatados neste estudo, cinco tiveram como sujeitos crianças. Dois foram realizados com adultos e dois analisaram o desempenho de estudantes no atendimento clínico, demonstrando a experiência de ensino do psicodiagnóstico interventivo psicanalítico, na prática de estágio, com estudantes de psicologia.

Com base nos dados coletados, os resultados serão apresentados em três categorias: psicodiagnóstico interventivo psicanalítico da criança; psicodiagnóstico interventivo psicanalítico do adulto; e psicodiagnóstico interventivo psicanalítico e formação profissional.

\section{PSICODIAGNÓSTICO INTERVENTIVO PSICANALÍTICO DA CRIANÇA}

Em um dos estudos com crianças que apresentavam queixa de comportamentos antissociais, Barbieri et al. (2004) investigaram se o psicodiagnóstico interventivo poderia contribuir para o tratamento da tendência antissocial. Foram analisadas oito crianças, entre cinco e dez anos de idade. Utilizaram-se entrevistas de anamnese, a Técnica de Rorschach, duas sessões lúdicas, entrevista familiar diagnóstica, Bateria Gráfica de Hummer, Teste de Apercepção Infantil (CAT-A), entrevistas devolutivas e follow-up com os pais. Durante o processo de avaliação foram incluídos assinalamentos e interpretações por parte do examinador. Foi realizado o follow-up por meio de entrevistas por telefone ou pessoalmente, no período de três a oito meses. Dos oito casos atendidos, sete foram concluídos. Dos sete concluídos cinco obtiveram sucesso terapêutico, todos com estrutura de personalidade neurótica e dois obtiveram fracasso terapêutico, com estrutura de personalidade psicótica. Os autores, embasados na análise dos dados do psicograma da Técnica de Rorschach, concluíram que as crianças com estrutura de personalidade neurótica puderam se beneficiar com o psicodiagnóstico interventivo, portanto, das sete crianças avaliadas, cinco se beneficiaram.

Interessados, ainda, nos resultados práticos do psicodiagnóstico interventivo, Barbieri et al. (2007) fizeram um estudo sobre o psicodiagnóstico interventivo no tratamento infantil, enfocando os fundamentos teóricos e a prática clínica. Para isso, aplicaram o método diagnóstico/terapêutico em uma menina de dez anos (Beatriz). Utilizaram a entrevista de anamnese, o Teste de 
Rorschach, duas sessões lúdicas, Entrevista Familiar Diagnóstica, Bateria Gráfica de Hammer, Teste de Apercepção Temática Infantil (CAT-A) e a entrevista devolutiva. A paciente foi encaminhada para atendimento por ter furtado dinheiro dos familiares em três ocasiões. Também foi salientada a obesidade da menina (52 kg), embora a queixa principal fossem os furtos. Beatriz vivia com o pai e a madrasta, foi descrita como sendo dócil e carinhosa, mas demonstrando dificuldade em ser contrariada, embora aceitasse bem os castigos infligidos pelos pais. Todo o psicodiagnóstico da paciente foi realizado de forma interventiva. Durante os seis meses seguintes ao tratamento, foram realizados três encontros com os pais, um a cada dois meses para se fazer o follow-up. Pode-se constatar que nesse período Beatriz não voltou a furtar. Com a utilização do psicodiagnóstico interventivo observou-se que após o contato com a psicóloga, Beatriz pode perceber mais claramente o que precisava, podendo, agora, buscar suas necessidades de modo simbólico e socialmente aceito. A conclusão dos autores foi a de que o psicodiagnóstico interventivo possibilitou uma avaliação mais fidedigna e fortaleceu o desenvolvimento tanto da menina, quanto da família em direção à cura. Portanto, os autores acreditam tratar-se "de um método promissor, que atribui ao paciente o papel de sujeito ativo ao alcance da própria saúde mental" (Barbieri et al., 2007, p. 181).

No atendimento infantil, o brincar para a criança é a forma que ela encontra de expressar naturalmente seus sentimentos e desejos (Silva, 2010). Quando o psicólogo interpreta o significado da brincadeira, mostra a situação em que se encontra a criança. Paulo (2009) realizou um estudo que teve como objetivo abordar o psicodiagnóstico interventivo com a técnica da hora de jogo para conhecer a realidade da criança, a técnica de investigação e a possibilidade de intervenção. A autora ilustrou seu trabalho com dois casos de atendimentos infantis, em que utilizou testes e a hora de jogo diagnóstica de forma interventiva. Utilizou também a entrevista compartilhada com pais e a criança. $O$ primeiro caso foi de um menino, de dois anos e seis meses, com queixa de hiperatividade e agressividade. O psicodiagnóstico foi realizado durante três encontros com a mãe e a criança juntas, nas quais foram feitas intervenções durante o processo. O segundo caso foi o de um menino de três anos, em que a queixa foi de fobia escolar. Realizou-se uma primeira entrevista somente com os pais e outra com os pais e a criança conjuntamente. Também foram feitas intervenções durante todo o processo. A autora concluiu que os resultados 
encontrados na prática clínica, têm revelado que a utilização da hora de jogo em entrevistas compartilhadas com pais e filhos, em 'triagem interventiva', 'plantão psicológico' e 'psicodiagnóstico interventivo', tem apresentado êxito, de forma que contribui para abreviar o psicodiagnóstico e diluir a barreira entre diagnóstico e terapia.

Barbieri, Jacquemin e Alves (2005) realizaram um estudo, em que a proposta foi verificar se as características de personalidade das mães de crianças, submetidas ao psicodiagnóstico interventivo, poderiam interferir nos resultados terapêuticos de seus filhos. Além disso, procurou-se determinar os prós e os contras desse novo método de avaliação, levando-se em consideração a personalidade materna. Participaram do estudo seis mães de sete crianças (de cinco a dez anos) que foram submetidas ao psicodiagnóstico interventivo em virtude de comportamentos antissociais. Todas as mulheres eram casadas, com idade entre 27 e 48 anos. Utilizou-se para a avaliação das mães o Teste de Rorschach, cujos resultados foram comparados com os das crianças que foram avaliadas com as entrevistas de follow-up. Foram considerados bem sucedidos os tratamentos em que, no follow-up os pais relataram melhora dos sintomas dos filhos. Os resultados do estudo com as mães mostraram que a mãe deprimida também poderia ser considerada como 'suficientemente boa'. E que uma mãe que tenha capacidade de identificação com o filho, mesmo que sua organização de personalidade seja pouco evoluída, pode ajudar melhor numa intervenção terapêutica, que uma mãe com personalidade neurótica, mas que é incapaz de estabelecer vínculos.

Assim como a criança necessita de uma mãe suficientemente boa, também necessita de um pai suficientemente bom. Outeiral (1997) acredita que o pai é aquele considerado pela criança como indestrutível, que faz com que esta se sinta segura, e mantenha seus 'impulsos livres e agressivos', e assim possa desfrutar destes à medida que tem um pai com capacidade de dizer 'não'. De acordo com essa perspectiva, Barbieri e Pavelqueires (2012) realizaram um estudo com o objetivo de verificar as características de personalidade dos pais (masculinos) de meninos e meninas com comportamentos antissociais, que foram avaliadas com o método do psicodiagnóstico interventivo, momento em que foram feitos apontamentos e interpretações. Participaram seis pais de sete crianças com idade entre cinco e dez anos, com faixa etária entre 26 e 56 anos. Utilizou-se o Teste de Rorschach para a avaliação desses pais, que foi aplicado 
individualmente após a entrevista de anamnese. Com a análise dos resultados percebeu-se que para o pai ajudar o filho, durante o psicodiagnóstico interventivo, teria que ser capaz de utilizar os conteúdos adquiridos por meio de seu envolvimento com a realidade externa e assim coibir, graduar e organizar as próprias pulsões e as angústias delas decorrentes. O estudo revelou que a figura paterna dessas crianças com comportamento antissocial, não se destacou com características suficientes para ajudá-las no tratamento, pois os pais mostraram muita dificuldade em dar-Ihes forma e limites, restando-Ihes a identificação narcísica com a angústia dessas crianças.

A partir destas considerações, verifica-se que o psicodiagnóstico interventivo pode ser utilizado por psicólogos no atendimento a crianças, pois auxilia o profissional na compreensão psicodinâmica do paciente e favorece intervenções durante o processo avaliativo, permitindo a evolução do quadro desde as consultas iniciais. Ao analisar os estudos citados destacam-se como limitações que algumas variáveis foram avaliadas apenas segundo o relato da mãe ou do pai, o que pode ter restringido a análise das informações levantadas nas entrevistas. Ainda, fazem-se necessários trabalhos que investiguem as interações entre as variáveis ambientais e pessoais, tais como as características da personalidade dos genitores com os resultados terapêuticos da criança simultaneamente, o que possibilitaria uma compreensão mais holística e íntegra a respeito das indicações e contra-indicações do Psicodiagnóstico Interventivo, uma vez que a dinâmica familiar pode exercer influência sobre o prognóstico infantil. A seguir, abordaremos os resultados desta modalidade de psicodiagnóstico no atendimento de adultos.

\section{PSICODIAGNÓSTICO INTERVENTIVO PSICANALÍTICO DO ADULTO}

Alguns profissionais, tais como Paulo (2006), Mishima e Barbieri (2009), estudaram a aplicação e os resultados do psicodiagnóstico interventivo no atendimento de adultos. A pesquisa desenvolvida por Paulo (2006) teve como objetivo verificar a possibilidade de se usar o modelo do psicodiagnóstico interventivo com pacientes adultos depressivos. Esse estudo foi realizado com quatro pacientes, dois homens e duas mulheres, com idade entre 31 e 57 anos, com diagnóstico de depressão. Teve duração de três meses, e foram utilizadas técnicas projetivas como facilitadores do contato entre terapeuta e paciente. As intervenções foram realizadas durante o processo, o mais precocemente 
possível. Os resultados encontrados foram satisfatórios, com uma melhora dos sintomas de depressão nos pacientes, em comparação com o início do tratamento. A conclusão da autora foi a de que o psicodiagnóstico interventivo, com a utilização de testes projetivos, trouxe maior facilidade de ocorrência de associações livres, dando mais espaço para o trabalho interpretativo.

Segundo Paulo (2006), as intervenções podem ser feitas já no início do psicodiagnóstico, e é preciso que o psicólogo se mantenha atento para apreender o material significativo que emerge durante a sessão terapêutica. Com as técnicas projetivas como ferramenta, o psicólogo, fará as intervenções, de forma a dar significado à vivência do paciente. Já está se fazendo o psicodiagnóstico interventivo no momento em que a postura do psicólogo e a utilização dos instrumentos, durante o procedimento, influenciam a reação emocional do sujeito, pois a simples introdução de um teste projetivo interfere nas experiências de vida e nas expectativas do paciente em relação àquele processo em andamento (Paulo, 2006). O psicodiagnóstico interventivo não tem como objetivo extinguir os sintomas, porém, possibilita uma avaliação mais completa, em que o paciente é fortalecido e preparado para uma psicoterapia, se for necessária (Tardivo, 2007).

Segundo Mishima e Barbieri (2009) e Barbieri (2008; 2009), no psicodiagnóstico interventivo, os instrumentos de avaliação são subordinados a métodos que não são estruturados, como, por exemplo, as técnicas projetivas, que são usadas como estratégias para a comunicação entre o psicólogo e o paciente. Essa pouca estruturação, somada às atitudes do profissional e acrescidas de sua maleabilidade psíquica, proporcionam a percepção e organização de dados importantes e significativos que o paciente apresenta durante o psicodiagnóstico interventivo. Portanto, as técnicas projetivas promovem um resultado diagnóstico mais seguro e fidedigno, possibilitando se verificar as mudanças que o psicodiagnóstico interventivo provoca na personalidade e quais pacientes possuem as características que proporcionariam benefícios com o uso do mesmo.

Mishima e Barbieri (2009) descreveram os resultados de um estudo em que os testes projetivos foram utilizados para o desenvolvimento do psicodiagnóstico interventivo. $O$ estudo teve como objetivo propiciar um tratamento psicoterápico para mulheres obesas, por meio do psicodiagnóstico interventivo, para verificar se uma abordagem de terapia breve provocaria mudanças no comportamento 
alimentar e o seguimento de uma dieta. O estudo foi realizado com uma paciente (Ana), com obesidade grau I (IMC $\left.=32 \mathrm{~kg} / \mathrm{m}^{2}\right)$, com 32 anos, com experiência em dietas de emagrecimento e exercícios físicos. Foram realizadas seis sessões de avaliação, nas quais se utilizou entrevista clínica semi-estruturada e testes projetivos com enfoque interventivo. A análise e interpretação dos dados foram feitas de acordo com o enfoque psicanalítico no momento da aplicação da entrevista e dos testes projetivos. Na análise dos resultados, considerou-se que a paciente apresentou uma estrutura de personalidade borderline. As autoras concluíram que após o atendimento com o psicodiagnóstico interventivo, Ana perdeu peso, chegando ao Índice de Massa Corporal (IMC) saudável. Mishima e Barbieri (2009) justificam esse resultado como sendo devido ao atendimento em que foi oferecido, durante o psicodiagnóstico interventivo, um holding e um ambiente suficientemente bom, que possibilitou a recuperação da capacidade criativa da paciente.

Desta forma, é importante, no psicodiagnóstico interventivo, "a adaptação ativa do analista às necessidades e expectativas do paciente [...] e, se necessária, a comunicação verbal desse entendimento no momento adequado" (Lescovar, 2004, p. 04), por meio do "uso de assinalamentos, holding, handling e interpretações" (Barbieri, 2008, p. 580). Assim, o psicodiagnóstico interventivo realizado no atendimento de adultos, deve proporcionar benefícios terapêuticos ao paciente, visando à diminuição de seu sofrimento psíquico desde as primeiras entrevistas.

Na análise dos artigos, é importante ressaltar que algumas particularidades limitam a generalização dos achados. Os estudos que abordam 0 psicodiagnóstico interventivo realizado com adulto tratam de uma amostra pequena, o que dificulta uma visão holística e mais fidedigna a respeito da eficácia desta técnica. Desta forma, sugerem-se novos estudos sobre o psicodiagnóstico interventivo com adultos, levando em consideração a diversidade de mecanismos psíquicos em funcionamento na personalidade, a fim de alcançar um aprofundamento na compreensão das aplicações deste método na prática clínica psicanalítica. 


\section{PSICODIAGNÓSTICO INTERVENTIVO PSICANALÍTICO E FORMAÇÃO PROFISSIONAL}

O aprendizado das intervenções fundamentais à atividade clínica do psicólogo deve ser iniciado na graduação, com o uso de novas ferramentas, como é o caso do psicodiagnóstico interventivo, que vem sendo utilizado por professores e alunos em estudos empíricos.

Becker (2002) realizou um estudo, com base na teoria Winnicottiana, com o objetivo de examinar como se dá o processo de acolhimento do paciente no primeiro atendimento efetuado pelo estagiário de psicologia. A autora destacou, em relação à prática nos atendimentos realizados por seus estagiários, que o psicodiagnóstico interventivo deve se caracterizar como um processo de maternagem, possibilitando um ambiente facilitador para a sucessão de fenômenos transicionais e permitiu a obtenção da compreensão diagnóstica.

Winnicott (1984), afirma que existe no momento das primeiras entrevistas um intercâmbio de comunicação mais livre entre o profissional e seu paciente do que na psicoterapia propriamente dita e fica muito difícil para o terapeuta, que avalia o caminho emocional do paciente, passar um longo tempo sem interpretar para só depois usar esse material. Segundo o autor, "não há nada mais difícil do que avaliar o caminho em que alguém se encontra sem fazer interpretação alguma [...] e depois, a certa altura, usar o material para interpretação do inconsciente" (Winnicott, 1984, p. 17).

Tardivo (2007) mostrou em seu trabalho realizado com estudantes de psicologia que o psicodiagnóstico interventivo é uma proposta que traz benefícios para o paciente. O objetivo do estudo foi verificar "se estudantes de graduação podem aprender a trabalhar segundo o modelo do psicodiagnóstico interventivo e atuar profissionalmente com base nas reflexões que podem e devem ser feitas 0 tempo todo" (Tardivo, 2007, p. 131). Um dos casos atendidos pelos estudantes e citado nesse estudo foi o de um menino, Leandro, 12 anos, órfão de mãe. A mãe foi assassinada pelo pai, que tentou suicídio. O pai foi aposentado, por problemas de saúde devido ao atentado. O paciente morava com o avô paterno e chegou à clínica com queixas de problemas de comportamento na escola. Em entrevistas, as tias maternas relataram mudanças de comportamento após a morte da mãe (medo de ficar sozinho). Durante os atendimentos Leandro demonstrou gostar da estagiária. Nas sessões foram realizadas intervenções que levaram a modificações importantes no comportamento do paciente, caracterizando-se o 
psicodiagnóstico interventivo. A estagiária, que foi supervisionada pela autora do artigo, pode observar, com o psicodiagnóstico interventivo, a ocorrência de mudanças internas em Leandro quanto às atitudes ao longo dos atendimentos; e também mudanças externas, em que precisou ter suas necessidades atendidas para poder crescer e se desenvolver. A supervisora (autora) chegou à conclusão de que esse tipo de psicodiagnóstico "é uma forma de atuação prática fundamentada que pode ser desenvolvida junto a estudantes de psicologia" (Tardivo, 2007, p. 134).

De acordo com Barbieri (2009), se levarmos em consideração os pontos comuns entre a psicanálise e o psicodiagnóstico interventivo, sobressai-se a dificuldade em separar a investigação da intervenção. A integração entre essas duas vertentes possibilita ao paciente se apropriar do que nele existe de mais criativo. Assim "a investigação psicanalítica pode ser transposta para qualquer situação em que exista um processo de associação livre" (Barbieri, 2009, p. 07). A autora ainda afirma que o psicanalista deve procurar muitas explicações para "eventos únicos" e, depois de integrá-las, organizá-las, e então usá-las nas intervenções direcionadas ao paciente. No psicodiagnóstico interventivo o objetivo é compreender profundamente o paciente, e estabelecer relações que façam o indivíduo repensar. No entanto, o terapeuta tem que ter o cuidado em usar os manejos adequados para que uma comunicação significativa se torne possível. É interessante que o profissional saiba ouvir o paciente, respeitando o seu ritmo e suas características, levando em conta a situação de contato em que terapeuta e paciente se encontram durante o psicodiagnóstico terapêutico (Lescovar, 2004).

Segundo Winnicott (1984), o trabalho realizado numa primeira entrevista pode servir como uma preparação para um tratamento mais demorado e intenso. Outras vezes, o resultado será oferecer à criança a possibilidade de ser compreendida e ajudada. No entanto "se uma criança sai da consulta terapêutica e retorna para uma situação familiar anormal ou social anormal, então não há provisão ambiental alguma da espécie necessária" (Winnicott, 1984, p. 13). Winnicott (1965/1993) citado por Barbieri (2008), alerta para o fato de que, se nas primeiras entrevistas não são feitas intervenções, os objetivos diagnósticos, além de não serem alcançados, impossibilitarão o contato terapêutico entre paciente e psicólogo. 
De acordo com Barbieri (2010), o psicólogo representa o elemento mais importante dos processos de avaliação e de intervenção. Ao invés dele fazer um trabalho solitário, pode contar com a participação do paciente nesse processo de intervenção, de modo a tornar esse trabalho confiável, além de obter ajuda para a seleção do material clínico. Assim, se faz possível a expansão das ações e o estímulo para transformações internas. Conforme alerta Tardivo (2007), o psicodiagnóstico interventivo vem se consolidando, gradativamente, como uma nova vertente, em que cada vez mais se valoriza 'a relação entre sujeitos'.

A análise dos artigos permitiu identificar que o número de publicações relativas ao aprendizado do psicodiagnóstico interventivo durante a graduação ainda é pequeno. Portanto, são necessárias outras pesquisas que averiguem os efeitos desse tipo de técnica em trabalhos desenvolvidos com crianças e adolescentes, adultos e idosos, enfocando os diferentes tipos de adoecimento mental e, deste modo, possibilitem uma compreensão profunda sobre a importância do aprendizado deste método ainda na graduação.

\section{DISCUSSÃO}

Os estudos analisados mostraram que é possível, durante o processo avaliativo, favorecer a possibilidade de associações livres, com apontamentos e interpretações que promovem a melhora dos sintomas apresentados pelos pacientes. As técnicas projetivas usadas em alguns dos estudos foram ferramentas que também auxiliaram na ocorrência de associações livres e ofereceram espaço para o trabalho interpretativo. Tais técnicas levam o paciente a se defrontar com suas fantasias e ter a possibilidade de, intuitivamente, ter uma melhor compreensão de si mesmo. Portanto, o psicodiagnóstico interventivo de orientação psicanalítica ajuda o paciente a se preparar para um processo psicoterápico e contribui para este trilhar o caminho em direção à promoção de sua saúde mental e, em alguns casos bem sucedidos, substituir uma longa psicoterapia.

A literatura também apresenta trabalhos realizados com alunos $\mathrm{e}$ estagiários do curso de psicologia, os quais evidenciaram que o psicodiagnóstico interventivo psicanalítico pode ser uma nova ferramenta para esses futuros profissionais, sinalizando outras possibilidades de atuação que diferem do modelo tradicional e que permitem um melhor acolhimento à subjetividade do paciente em sofrimento psíquico. 
Pode-se afirmar que esse método provoca no paciente a possibilidade de ser sujeito ativo de seu próprio caminho em direção à sua saúde mental e possibilita ao psicólogo realizar uma avaliação mais fidedigna e globalizada. E é confiando no potencial do ser humano e na existência do inconsciente, que o profissional se permite ocupar o lugar de 'objeto-subjetivo' de seu paciente e assim poder estar em íntimo contato com ele. Portanto, o psicodiagnóstico interventivo psicanalítico é um método que pode promover benefícios terapêuticos por meio da compreensão intuitiva do inconsciente, em que o paciente tem a possibilidade de, por meio de um holding bem estabelecido, num ambiente suficientemente bom, diminuir seu sofrimento mental e se reorganizar.

Uma das limitações, para este estudo, foi a escassez de publicações sobre o psicodiagnóstico interventivo com abordagem psicanalítica. Considera-se que seria importante dimensionar os efeitos desse tipo de técnica em trabalhos desenvolvidos com crianças e adolescentes, adultos e idosos, enfocando os diferentes tipos de transtornos e as intervenções mais pertinentes mediante a queixa apresentada. Os resultados favoráveis mostrados pelos estudos relatados reiteram a importância do aprofundamento das pesquisas sobre o tema, que é um terreno fértil para futuras investigações, já que o psicodiagnóstico interventivo psicanalítico deve estar em constante processo de evolução.

\section{REFERÊNCIAS}

Ancona-Lopez, M. (1998). Psicodiagnóstico: Processo de intervenção. São Paulo: Cortez.

Barbieri, V. (2008). Por uma ciência-profissão: O psicodiagnóstico interventivo como método de investigação científica. Psicologia em Estudo, 13(3), 575584.

Barbieri, V. (2009). O psicodiagnóstico interventivo psicanalítico na pesquisa acadêmica: Fundamentos teóricos, científicos e éticos. Boletim de Psicologia, 59(131), 209-222.

Barbieri, V. (2010). Psicodiagnóstico tradicional e interventivo: Confronto de paradigmas? Psicologia: Teoria e Pesquisa, 26(3), 505-513.

Barbieri, V., \& Pavelqueires, J. G. (2012). Personalidade paterna como fator prognóstico no tratamento da tendência antissocial. Paidéia (Ribeirão Preto), 22(51), 101-110. 
Barbieri, V., Jacquemin, A., \& Alves, Z. M. M. B. (2004). Alcances e limites do psicodiagnóstico interventivo no tratamento de crianças anti-sociais. Paidéia (Ribeirão Preto), 14(28), 153-167.

Barbieri, V., Jacquemin, A., \& Alves, Z. M. M. B. (2005). Personalidade materna e resultados de crianças no psicodiagnóstico interventivo: 0 que significa 'mãe suficientemente boa'? Psico, (Porto Alegre), 36(2), 117-125.

Barbieri, V., Jacquemin, A., \& Alves, Z. M. M. B. (2007). O psicodiagnóstico interventivo como método terapêutico no tratamento infantil: Fundamentos teóricos e prática clínica. Psico (Porto Alegre), 38(2), 174-181.

Becker, E. (2002). Os bastidores de uma história: A expressão criativa de estagiários na prática do psicodiagnóstico interventivo. Tese de Doutorado, Instituto de Psicologia, Universidade de São Paulo, São Paulo.

Lescovar, G. Z. (2004). As consultas terapêuticas e a psicanálise de D. W. Winnicott. Estudos de Psicologia (Campinas), 21(2), 43-61.

Mishima, F. K. T.; Barbieri, V. (2009). Saúde feminina: Considerações sobre o psicodiagnóstico interventivo na obesidade. Mudanças-Psicologia da Saúde, $17(2), 92-100$.

Outeiral, J. (1997). Sobre a concepção de pai na obra de D. W. Winnicott. In I. F. M. Catafesta (Org.). A clínica e a pesquisa no final do século: Winnicott e a universidade (p. 91-104). São Paulo: Lemos.

Paulo, M. S. L. L. (2006). Psicodiagnóstico interventivo em pacientes adultos com depressão. Boletim de Psicologia, 56(125), 153-170.

Paulo, M. S. L. L. (2009). A importância da possibilidade de intervenção na hora do jogo diagnóstica. In Anais do I Congresso Brasileiro de Ludodiagnóstico (p.131-133). São Paulo: Escola Paulista de Psicologia Avançada.

Santiago, M. D. E. (1998). Psicodiagnóstico: Uma prática em crise ou uma prática na crise? In: M. Ancona-Lopez. Psicodiagnóstico: Processo de intervenção. São Paulo: Cortez.

Silva, M. C. P. (2010). A consulta terapêutica: Um espaço potencial para a construção da parentalidade. Jornal de Psicanálise, 43(79), 143-154

Tardivo, L. S. P. C. (2007). Psicodiagnóstico interventivo: Uma proposta de ensino em Atendimento Clínico. Mudanças - Psicologia da Saúde, 15(2), 128-134.

Trinca, W. (1998). Processo diagnóstico de tipo compreensivo. In: W. Trinca. Diagnóstico psicológico: A prática clínica. São Paulo: Cortez. 
Winnicott, D. W. (1984). Consultas terapêuticas em psiquiatria infantil. Rio de Janeiro: Imago.

Contato: rute.milani@unicesumar.edu.br, merces.tom@gmail.com, brunamilhorini@hotmail.com

Recebido em: 09/04/2014

Revisado em: 19/05/2014

Aceito em: 15/07/2014 